\title{
Practice of Distance Online Teaching of Automobile Theory
}

\section{Yongfang Li, Ruoping Zhang, Yali Yang}

School of Mechanical and Automotive Engineering, Shanghai University of Engineering Science, Shanghai, China Email: muzicandy@126.com

How to cite this paper: Li, Y.F., Zhang, R.P. and Yang, Y.L. (2021) Practice of Distance Online Teaching of Automobile Theory. Open Access Library Journal, 8: 66918. https://doi.org/10.4236/oalib.1106918

Received: October 21, 2020

Accepted: January 1, 2021

Published: January 4, 2021

Copyright $\odot 2021$ by author(s) and Open Access Library Inc.

This work is licensed under the Creative Commons Attribution International License (CC BY 4.0).

http://creativecommons.org/licenses/by/4.0/

\begin{abstract}
The paper introduces the online teaching mode of automobile theory course, which is based on the Chaoxing course platform, the interaction between teachers and students by QQ group classroom platform, and online supervision of final examination in Tencent meeting. The paper explores and practices the course online teaching. This online teaching practice not only improves students' autonomous learning ability, but also achieves the teaching effect required by the syllabus in teaching methods, academic performance and management mode.
\end{abstract}

\section{Subject Areas}

Education

\section{Keywords}

Automobile Theory, Network Teaching, Online Teaching

\section{1. 研究背景}

随着计算机技术的大力发展和网络的普及应用, 远程网络教学成为一种 新兴的教学模式, 越来越受到关注。网络教学平台为远程教学的学习者和教 育者提供了有力的支持工具。平台上可以进行授课、学习、互动、作业处理 以及期末考核等教学过程。近年来, 各高等院校对网络教学平台的应用逐渐 普及, 通过开放在线课程, 建设精品课程等方式, 拓展了教学时空、更新了 教学手段, 充分利用了教学资源, 激发学习者学习的积极性和主动性[1]。特 别是近期受到新型冠状病毒肺炎疫情的影响, 各大高校都开展了远程在线教 育。这种教学模式对课程的教学方法、教学管理体制、教学过程组织和最终 考核方式等教学过程提出了很高的挑战, 这也为高等教育教学改革与发展带 来新的机遇和挑战。 


\section{2. 线上授课模式}

汽车理论是汽车类专业人才培养的专业必修课程。我校汽车理论教学组 的教师根据课程实际情况, 制定并开展了汽车理论课程线上授课模式。超星 课程平台在课程管理方面具有突出优势, 但直播答疑方面经常会出现网络卡, 所以不建议直接用超星平台进行直播答疑。QQ 群课堂虽然不便于学生日常 的管理, 但利用 $\mathrm{QQ}$ 群课堂老师可以随时和学生交流以及直播答疑。腾讯会 议也是很不错的授课平台, 但限于腾讯会议不好发送图片, 不好保存交流记 录等, 腾讯会议更适合用在在线监考上。因此, 本次在线教学实践模式包括 利用超星课程平台进行课程管理, 利用 $\mathrm{QQ}$ 群课堂进行直播答疑互动以及利 用腾讯会议进行在线监控考核的组合式线上混合教学模式, 综合发挥各平台 的凸显功能, 优化线上教学模式, 提高线上教学效率。超星课程平台即 “超 星学习通”, 是一款面向手机、平板、电脑等多个移动终端的专业学习平台, 作为新一代网络教学工具, 它具有课堂签到、调查投票、分组讨论、抢答提 问等多种功能[2]。这种组合式线上混合教学模式改变了传统的 “灌输式” 教 育模式, 重塑了课堂教学活动。因此, 汽车理论课程远程在线教学实践对下 一步合理应用网络教学工具, 构建先进的专业课程教学模式, 提高汽车理论 课程的教学质量有意义重大。

\section{3. 课程教学设计}

\section{1. 教学内容设计}

汽车理论课程的教学内容主要包括汽车的动力性、燃油经济性、制动性、 操纵稳定性、平顺性、通过性等各使用性能的评价指标与评价方法。课程要 求学生学会建立有关动力学方程, 在理解的基础上分析汽车及其零部件的结 构形式与结构参数对各项使用性能的影响, 掌握汽车使用性能预测的基本计 算方法。

每个章节指定教学目标, 明确学习的基本要求和教学的重点难点。下面 以第一章汽车的动力性为例, 指定该章前三小节的教学目标。第一节, 汽车 的动力性指标, 掌握汽车动力性的定义, 了解汽车动力性的理论分析过程。 掌握汽车动力性的评定指标, 理解各评定指标的含义, 了解汽车动力性指标 的测定条件与一般数值。第二节, 汽车的驱动力与行驶阻力, 理解汽车驱动 力的定义与计算公式, 理解驱动力计算公式中各参数的含义, 了解驱动力的 影响因素; 理解汽车驱动力图的含义。理解滚动阻力的成因, 会进行弹性车 轮在硬路面上滚动时的受力分析, 理解路面对驱动轮的切向反作用力与驱动 力之间的区别; 理解滚动阻力系数的含义, 了解滚动阻力系数的影响因素, 知道典型路面滚动阻力系数的数值。理解空气阻力的含义与构成; 理解空气 阻力系数的含义, 了解空气阻力系数的影响因素, 知道空气阻力系数的典型 数值。理解坡度阻力的含义。理解道路阻力的含义。理解加速阻力的含义, 理解旋转质量换算系数的含义。能够熟练地运用各阻力计算公式进行计算。 第三节, 汽车的驱动力-行驶阻力平衡图, 掌握汽车行驶方程式, 理解驱动力 -行驶阻力平衡图的含义, 掌握驱动力-行驶阻力平衡图的绘制方法, 能够熟 
练地绘制驱动力-行驶阻力平衡图。掌握运用汽车行驶方程式和驱动力-行驶 阻力平衡图确定各动力性指标的方法; 能够灵活运用汽车行驶方程式和驱动 力-行驶阻力平衡图确定各动力性指标和进行相关的计算分析。理解动力特性 图的含义, 掌握动力特性图的绘制方法, 能够运用动力特性图确定各动力性 指标。通过明确每一小节的每一个知识点的教学目标, 教师就可以有针对性 的进行知识点教学, 学生也更容易理解并掌握相应知识点。

\section{2. 教学方式设计}

1) 课前准备

学校对全校授课教师进行了超星平台的培训, 让教师熟悉该平台的使用。 该平台上有课程管理模块, 教师首先进行团队管理, 把课程组的老师全部放 进去, 这样大家可以共享教学资源, 不需要重复上传, 然后按照班级建立上 课班级学生名单 [3]。最后课程组教师进行建课, 把课程教学大纲和各章节的 教学相关资料上传。教学相关资料包括电子教材、授课 PPT、授课视频、试 题库、习题库以及课后作业等等 $[4]$ 。授课视频都是短视频, 按照知识点, 分 配给汽车理论课程组各位老师自己录制。争取一个知识点对于录制一个小视 频, 所以单个小视频的时间可能几分钟至十几分钟, 避免了连续长时间授课 带来的单调和枯燥, 以更好的提高学生的学习兴趣。同时, 这样授课就相当 于虽然是一门课程, 学生却能观看课程组所有老师不同知识点的授课视频, 这是传统教室上课所不能达到的。

除了超星平台外, 上课前授课教师申请一个 $\mathrm{QQ}$ 群, 全班的同学加入该 QQ 群, 方便教师辅助性的发布一些信息, 并随时与学生进行交流。同时, 通过该群教师可以直接进入 $Q Q$ 群课堂给学生进行现场直播讲解和讨论。

每次正式上课前 20 分钟, 教师可以在超星平台设置上课提醒, 并提前 5 分钟发布签到活动, 学生在课前收到签到通知进行签到然后进入超星平台进 行学习。

2) 课堂学习

由于课堂学习是在线形式, 每次课堂学习主要包括三个阶段: 教师发布 任务阶段、学生自主学习阶段和教师总结阶段 [3]。第一阶段(约 5 分钟): 教 师发布任务, 可以在 $\mathrm{QQ}$ 群里直接发布, 也可以通过 $\mathrm{QQ}$ 群课堂直播平台进 行发布。主要是引入本次课程新知识, 学生需要学习的知识点, 以及超星平 台上需要完成的任务点。第二阶段(约 30 分钟): 学生自主学习阶段, 学生主 要是完成超星平台的各个任务点, 包括课程 P P T 内容、课堂知识点小视频、 随堂作业、讨论话题等。教师可以在超星课程平台观察学生各个任务点完成 情况, 统计各知识点学生掌握情况, 对突出问题、共性问题等存在问题做好 记录。对没有按时完成任务点的同学进行提醒, 对作业没有按要求完成的同 学以打回的方式让同学重新完成。第三阶段(约 10 分钟): 回到 $Q Q$ 群课堂直 播平台进行课程总结、重点难点讲解、给学生答疑、主题讨论互动等。

3) 课后作业

教师在超星课程平台发布课后作业, 主题讨论, 调查问卷以及相关知识 的拓展等。学生需在规定期限内完成这些课后作业以及其他一些相关任务。 
超星课程平台有个非常好的地方就是, 教师上传的所有课程资料都是在线保 存的, 学生有不懂或者有疑问的地方, 可以反复观看知识点的小视频以及 PPT 等教学资料, 这也是传统课堂教学不能实现的。在超星课程平台所有学生的 所有任务点的完成情况都有记录, 包括签到考勤、任务点的完成比例、作业、 课堂互动、课后作业、知识拓展以及主题讨论等, 教师可以通过数据统计很 容易且清楚的了解到, 因此可以通过数据统计分析, 关注每一位学生, 不让 任何学生掉队。

4) 课程考核

汽车理论课程的最终总评成绩是按照平时成绩和最终期末考试成绩两部 分组成, 两部分的比例按 3:7 组合而得。在超星课程平台上, 课程活动过程 的所有任务点列入平时考核成绩, 这个统计过程可以由平台自动完成。期末 考试成绩主要是在线考试完成。教师首先需要组好试题, 由于之前教师已经 在超星课程平台上传了试题库, 因此只需要用该平台自动组题功能完成期末 考试的组题工作, 设置好发放时间和结束时间, 提醒学生按时进入超星平台 领取试卷并在线作答, 结束后上传至该平台完成考试。为了实现在线监考, 教师需要提前申请腾讯会议, 考生提前进入考场并安装好摄像头, 教师核对 学生信息并进行在线监考直至考试结束。这个监考过程可以录屏, 也体现了 考试的公平公正。

\section{4. 课程总结分析}

我校利用该教学方法完成了汽车理论课程的在线教学。通过课程考核结 束后教师进行课程总结分析, 查考学生网上评教评分情况, 开展学生在线座 谈会, 同行教师的评价以及教师座谈会, 对本次授课情况做一个客观全面的 分析总结。本次汽车理论在线教学在授课内容、授课方式、学习成绩以及管 理模式等方面都达到了满意的结果, 学生对于这种教学模式也是非常认同, 同时也提高了学生自主学习的能力。

\section{5. 结语}

在线教育的优势很明显, 学生可以反复学习没有掌握的相关知识点, 学 习时间和地点也比较灵活, 可以随时随地的学习, 教师的教学资源可以共享, 减轻教师的工作量, 同时教师可以很方便地统计教学过程中的所有教学活动, 做到更加全面地评估教学过程中存在的问题以及学生对知识点的掌握情况, 并及时与学生互动和辅导。但在线教育毕竟是网络教育, 教师与学生以及学 生之间都是各自对着屏幕, 缺乏亲切感, 较难拉近师生之间以及同学之间的 距离。另外, 在线教育对学生自主学习提出了较高要求, 有极个别学生自主 学习能力很差, 这时教师的及时发现和沟通就显得很重要了。总之, 通过这 次的在线教学实践可以发现, 此类在线教学模式可以满足正常的教学秩序。 教学形式毕竟只是手段, 如何合理地将传统课堂教学和网络平台教学结合起 来, 进一步提升教学质量, 是值得当代教育工作者长期思考和探索的问题 [4]。 现在已经回归正常课堂教学, 通过这次在线教学的尝试和实践, 充分利用网 络手段丰富教学方式, 对于提升汽车理论课程的教育教学水平有重要意义。 


\section{Conflicts of Interest}

The authors declare no conflicts of interest regarding the publication of this paper.

\section{References}

[1] 方瑞莲, 赵海敬. 基于网络教学平台的线上授课教学活动设计浅析一一以《汽车 构造(下)》理论教学为例 [J]. 中外企业家, 2020(21): 168-169.

[2] 赵旭华, 赵虹, 翟翔, 等. 学习通平台在生物化学与分子生物学教学中的实践 [J]. 基础医学教育, 2019, 21(10): 830-832.

[3] 周慧芳. 基于超星学习通与钉钉平台的生理学教学实践 [J]. 卫生职业教育, 2020, 38(19): 67-68.

[4] 刘峰舟, 王瀚, 张敏, 王小成, 薛军辉. 超星学习通在航空航天临床医学理论教 学中的应用研究[J]. 医学教育研究与实践, 2020, 28(5): 820-824.

\section{Appendix (Abstract and Keywords in Chinese)}

\section{汽车理论课程远程在线教学实践}

摘要: 对汽车理论课程采用了基于超星课程平台的教学资源网络化, 利用 $\mathrm{QQ}$ 群课堂平台进行师生互动以及采用腾讯会议进行期末考试的在线监考等 在线教学模式, 对该课程网络教学进行了探索和实践。本次在线教学实践不 但提高了学生自主学习的能力, 在授课方式、学习成绩和管理模式等方面都 达到教学大纲所要求的教学效果。

关键词: 汽车理论; 网络教学; 在线教学 